OPEN ACCESS

Edited by:

Herbert Jelinek,

Charles Sturt University, Australia

Reviewed by:

Radu liescu,

Grigore T. Popa University of Medicine and Pharmacy, Romania Sarah S. Knox,

West Virginia University School of Medicine, USA

*Correspondence:

Danuta Makowiec, Institute of Theoretical Physics and Astrophysics, University of Gdańsk, ul. Wita Stwosza 57, Gdańsk 80-952,

Poland

fizdm@ug.edu.pl; Zbigniew R. Struzik,

Graduate School of Education, The University of Tokyo, 7-3-1 Hongo, Bunkyo-ku, Tokyo 113-0033, Japan

z.r.struzik@p.u-tokyo.ac.jp

Specialty section: This article was submitted to

Computational Physiology and Medicine, a section of the journal Frontiers in Physiology

Received: 30 December 2014 Accepted: 16 June 2015

Published: 14 July 2015

Citation:

Makowiec D, Wejer D, Kaczkowska A Żarczyńska-Buchowiecka $M$ and Struzik ZR (2015) Chronographic imprint of age-induced alterations in heart rate dynamical organization. Front. Physiol. 6:201. doi: 10.3389/fphys.2015.00201

\section{Chronographic imprint of age-induced alterations in heart rate dynamical organization}

\author{
Danuta Makowiec ${ }^{1 *}$, Dorota Wejer ${ }^{1}$, Agnieszka Kaczkowska ${ }^{2}$, \\ Marta Żarczyńska-Buchowiecka ${ }^{3}$ and Zbigniew R. Struzik ${ }^{1,4,5 *}$
}

${ }^{1}$ Institute of Theoretical Physics and Astrophysics, University of Gdańsk, Gdańsk, Poland, ${ }^{2}$ Faculty of Applied Physics and Mathematics, Gdańsk University of Technology, Gdańsk, Poland, ${ }^{3} 1$ st Chair \& Clinic of Cardiology, Medical University of Gdańsk, Gdańsk, Poland, ${ }^{4}$ RIKEN Brain Science Institute, Wako-shi, Japan, ${ }^{5}$ Graduate School of Education, The University of Tokyo, Tokyo, Japan

Beat-to-beat changes in the heart period are transformed into a network of increments between subsequent RR-intervals, which enables graphical descriptions of short-term heart period variability. Three types of such descriptions are considered: (1) network graphs arising from a set of vertices and directed edges, (2) contour plots of adjacency matrices $A$, representing the networks and transition matrices $T$, resulting from $A$, and (3) vector plots of gradients of the matrices $A$ and $T$. Two indices are considered which summarize properties of $A$ and $T$ : the approximate deceleration capacity and the entropy rate. The method, applied to time series of nocturnal RR-intervals recorded from healthy subjects of different ages, reveals important aspect of changes in the autonomic activity caused by biological aging. Independent of the subject's age, following accelerations, a pendulum-like dynamics appears. With decelerations, this dynamics develops in line with the subject's age. This aging transition can be graphically visualized by vectors connecting the maxima of the transition probabilities of $\mathrm{T}$, which, metaphorically, resemble a chronometer or the hands of a clock.

Keywords: short-term heart rate variability, aging, deceleration capacity, entropy rate

\section{Introduction}

The two branches of the autonomic nervous system (ANS), the sympathetic and vagal subsystems, cooperate to maintain homeostasis in the cardiovascular (CV) system, namely the continuous controlled movement of blood through the whole organism to transport nutrients to organ tissues to every cell of the organism (Guyton and Hall, 2006). With advancing age, a gradual impairment in the functioning of the complex interplay between these two subsystems develops (Esler et al., 1995). The phenomenon of such age-related ANS-CV deterioration is revealed as noticeable alterations in the cardiac interbeat RR-interval dynamics. Signals with RR-intervals derived from long-term ECG recordings have been widely used in assessing ANS responses during normal activities in health and disease (Reardon and Malik, 1996; Pikkujämsä et al., 1999; Crasset et al., 2001; Struzik et al., 2004, 2006; Shimazu et al., 2005; Beckers et al., 2006; Meersman and Stein, 2007; Monahan, 2007; Stein et al., 2009; Callegaro and Taylor, 2010; Makowiec et al., 2011). The reasons for the observed variations are not yet completely understood (Goldberger et al., 2008). Nevertheless, heart rate variability is a recognized surrogate index for cardiac autonomic function of the sinus node and ventricles, and is a marker of im-/balance in the sympathetic and vagal activities (Goldberger et al., 2008; Poirier, 2014). 
In particular, in Reardon and Malik (1996), a significant decrease with age was shown in indices of heart rate variability (HRV) based on an overall HRV estimation. But for indices which describe short-term components of HRV, and are therefore expected specifically to reflect vagal modulation, no significant change was observed. Struzik et al. (2006) reported a progressive alteration in the sympathetic/parasympathetic balance and put forward the "autonomic aging" hypothesis, involving emerging parasympathetic dominance. Alteration in the long-range organization, and a loss of complexity have been reported by Pikkujämsä et al. (1999), Beckers et al. (2006), and Viola et al. (2011), though fractal scale-invariance and nonlinear properties have been claimed to remain stable with the advance of age (Schmitt and Ivanov, 2007). Schmitt et al. (2009) further argued that a sleep phase-based, well-pronounced cardiac risk stratification pattern remains unchanged with age, even in elderly patients. Yet the elementary linear measures of autonomic activity are known to be strongly influenced by both the sleep phase structure and aging (Brandenberger et al., 2003) - apparently through altered respiratory patterns in the elderly.

The ECG data are of fixed resolution, which leads to the natural discretization of values for the RR-intervals. As a consequence, the values of changes in the RR-intervals: accelerations and decelerations also become multiples of the intrinsic signal resolution. Such quantification has an effect in setting up a state space of a finite size, the elements of which can consistently be labeled by values representing the RR-increments. As a consequence, a framework can be obtained, which is particularly suitable for analysis by methods based on the symbolic dynamics approach, including network representation (Donner et al., 2010; Campanharo et al., 2011). For example, one can ask whether the subsequent change in the RR-interval is related to the current change or not, and if so, what this relation looks like. Different mechanisms within the heart rate regulatory system are assumed to be responsible for accelerations and for decelerations (Guyton and Hall, 2006). Adjustments caused by the sympathetic system are slow, on a scale of seconds, whereas adjustments caused by the vagal system are fast (Stein and $\mathrm{Pu}, 2012$; Poirier, 2014). Therefore, qualification and quantification of changes in RRintervals provide insights into mechanisms driving the heartbeat dynamics.

In the following, we describe a geometrically inspired method, which enables the assessment of the dependencies between subsequent changes in the RR-intervals from two aspects: the visual aspect, due to which an intuitive description is given, and the quantitative aspect, which provides the rationale behind our argumentation. In particular, we apply the method to analyze beatto-beat variability of nocturnal heart periods of healthy people of different ages, from young people at the age of twenty to the elderly at ages of up to ninety. Two indices are considered to draw conclusions about the geometric properties observed. The entropy rate gives an overall measure of changes in the heard period resulting from the Markov chain representation. The deceleration capacity (Schumann et al., 2010) is tuned to be concentrated on properties of large decelerations, hence aims to quantify events in which the vagal activity is expected to be especially high.
This work is a continuation of our earlier investigations into the usability of tools from network theory in the assessment of RR-time interval signals (Makowiec et al., 2014, 2015a,b).

\section{Materials and Methods}

\subsection{Signal Preprocessing}

Twenty-four-hour Holter ECG recordings during a normal sleep-wake rhythm were obtained from healthy volunteers without any known cardiac history. Individuals with a high risk of obstructive sleep apnea [see Table 2 in Epstein et al. (2009)] were not included in the study. All the participants in the study gave their informed consent. The study complied with the Declaration of Helsinki and was approved by the Bioethics Committee of the Medical University of Gdańsk.

The participants, in total 194 people, were pooled into agedependent groups: twenties: 36 subjects (18 women, 18 men, age: 20-29), thirties: 26 subjects (13 women, 13 men, age: $30-39)$, forties: 36 subjects ( 16 women, 20 men, age: $40-49$ ), fifties: 32 subjects (13 women, 19 men, age: $50-59$ ), sixties: 24 subjects ( 11 women, 13 men, age: $60-69)$, seventies: 22 subjects (10 women, 12 men, age: 70-79), and eighties: 18 subjects (11 women, 7 men, age: $80-89$ ). Holter recordings were first analyzed using Del Mar Reynolds Impresario software and screened for premature, supraventricular and ventricular beats, missed beats, and pauses. Then, the signals were thoroughly corrected manually and annotated correspondingly. The sleep hours were selected for each signal individually, according to the day-night transition observed in the length of the RR-intervals. Finally, a six-h period, covering the longest RRintervals, was extracted. Perturbations in a signal (artifacts or any but normal-to-normal RR-intervals), consisting of less than five consecutive RR-intervals, were replaced by the medians calculated from the last seven normal RR-intervals. Other perturbations were deleted. Ultimately, the signals studied were constructed from at least 20,000 normal-to-normal RR-intervals. The Holter equipment used by us provided signals with $128 \mathrm{~Hz}$ sampling frequency, which sets the resolution of the RR-intervals to approximately $8 \mathrm{~ms}$. As a consequence, all the values obtained for RR-intervals, and in turn for RR-increments, are multiples of $8 \mathrm{~ms}$.

If $\mathbf{R} \mathbf{R}=\left\{R R_{0}, \ldots, R R_{i}, \ldots, R R_{N}\right\}$ is the time sequence of RR-intervals, $i$ is the time index, then the signal of RRincrements is defined as $\Delta \mathbf{R} \mathbf{R}=\left\{\delta R R_{1}, \ldots, \delta R R_{i}, \ldots, \delta R R_{N}\right\}$ with $\delta R R_{i}=R R_{i}-R R_{i-1}$. Hence, an event described by $\delta R R_{i}>0$ denotes a deceleration and each event characterized by $\delta R R_{i}<0$ is an acceleration. When $\delta R R_{i}=0$, we say a no-change event has taken place.

The state space of the signal values consists of a finite number of multiplies of $8 \mathrm{~ms}$, namely $0, \pm 8, \pm 16, \ldots$, ms which, when arranged from the smallest acceleration to the largest deceleration, are referred as the following set:

$$
\Delta_{J} \in\left\{-\Delta_{K}, \ldots, 0, \ldots, \Delta_{K}\right\}, \Delta_{K}=\max _{i}\left\{\left|\delta R R_{i}\right|\right\} .
$$

By applying binning to RR-intervals, e.g., with a bin equal to $48 \mathrm{~ms}$, the corresponding set of state space values (1) decreases, which allows for a more compact presentation of the results. Unless otherwise described, the results presented were obtained with the accuracy of the intrinsic signal resolution. 


\subsection{Assessment of RR-Increments}

In a sequence of pairs $\left\{\left(\delta R R_{i}, \delta R R_{i+1}\right), i=1, \ldots, N\right\}$, constructed from a given signal $\Delta \mathbf{R R}$, we search for all pairs $\left(\Delta_{I}, \Delta_{J}\right), I$, $J=-K, \ldots, K$, and then normalize the count of each pair to obtain the $(I, J)$-th element of a square matrix $\mathbf{A}$ :

$$
A_{I J}=\frac{\left|\left\{\left(\delta R R_{i}, \delta R R_{i+1}\right): \delta R R_{i}=\Delta_{I}, \delta R R_{i+1}=\Delta_{J}\right\}\right|}{N} .
$$

In this way, the matrix $\mathbf{A}$ contains probabilities that events $\left(\Delta_{I}\right.$, $\Delta_{J}$ ) occur one by one in a time sequence. Matrix $\mathbf{A}$ can be read as an adjacency matrix of a directed and weighted network with vertices defined by (1) and with directed edges leading from $\Delta_{I}$ to $\Delta_{J}$, the weight of which is given by $A_{I J}$.

Straightforward algebra ensures that:

$$
\begin{aligned}
& \frac{1}{4}\left(R R_{i}+R R_{i+1}-R R_{i-1}-R R_{i-2}\right) \\
& \quad=\frac{1}{4}\left(\delta R R_{i-1}+\delta R R_{i}+\delta R R_{i}+\delta R R_{i+1}\right),
\end{aligned}
$$

which means that the deceleration capacity (DC) obtained by the phase-rectified signal averaging method (PRSA) (Schumann et al., 2010) can be considered to be a function in the space of RR-increments. Moreover, we can use the general formula of DC for decelerations of only a given size, and in this way obtain the index of DC limited to the given decelerations. In particular, for some presumed deceleration size limit $\Delta_{D}$, information about decelerations and accelerations from the matrix $\mathbf{A}$ can be used to estimate the approximate DC based on the formula:

$$
\begin{aligned}
D C_{\mathbf{A}}= & \frac{1}{4}\left[\sum_{\Delta_{I} \geq \Delta_{D}} \sum_{\Delta_{K}}\left(\Delta_{K}+\Delta_{I}\right) A_{K I}\right. \\
& \left.+\sum_{\Delta_{I} \geq \Delta_{D}} \sum_{\Delta_{J}}\left(\Delta_{I}+\Delta_{J}\right) A_{I J}\right]
\end{aligned}
$$

In our further calculations, we assume $\Delta_{D}=40 \mathrm{~ms}$, because this value corresponds to a $5 \%$ change in the length of RRintervals.

For any adjacency matrix $\mathbf{A}$, a corresponding transition matrix $\mathbf{T}$ can be introduced as follows:

$$
T_{I J}=\frac{A_{I J}}{\sum_{J} A_{I J}}=P\left(\Delta_{J} \mid \Delta_{I}\right) .
$$

Matrix T describes the conditional probability of observing $\Delta_{J}$ if an increment $\Delta_{I}$ has taken place. Similarly to matrix $\mathbf{A}$, matrix $\mathbf{T}$ can also be represented as a directed and weighted network with the same vertices as for matrix $\mathbf{A}$, but with edges corresponding to the probabilities of transitions from a given vertex.

Transition matrix $\mathbf{T}$ captures and represents a Markov process, which underlies changes in the system studied. Matrix $\mathbf{T}$ is right stochastic, and consequently its stationary state can be inferred. This stationary state $\mu$ comprises the eigenvector of $\mathbf{T}$ corresponding to eigenvalue 1 . Consequently, we can calculate the entropy rate as follows:

$$
S_{T}=-\sum_{I=-K}^{K} \mu_{I} \sum_{J=-K}^{K} T_{I J} \ln T_{I J} .
$$

Finally, for each matrix $\mathbf{A}$ and $\mathbf{T}$, we can construct gradient matrices, GA and GT, respectively, consisting of vectors which describe the direction and power of local changes:

$$
\begin{aligned}
G A_{I J} & =\left(A_{I, J+1}-A_{I, J-1}, A_{I+1, J}-A_{I-1, J}\right) \\
G T_{I J} & =\left(T_{I, J+1}-T_{I, J-1}, T_{I+1, J}-T_{I-1, J}\right) .
\end{aligned}
$$

\section{Results}

In Figures 1 and 2, and Table 1, we show the results of the method applied to the data described. These plots show properties of the mean matrices $\mathbf{A}$ and $\mathbf{T}$ obtained by averaging matrices calculated for individual subjects and then pooled into the age groups. Unfortunately, graphs of networks constructed at the signals' resolution accuracy, namely $8 \mathrm{~ms}$, would be barely legible. Therefore, these networks are presented as contour plots of the adjacency and transition matrices; see the first rows of Figures $\mathbf{1}$ and 2. The plots of $\mathbf{A}$ describe the probability distributions of observing in a sequence $\Delta_{I}-$ shown on the horizontal axis, and $\Delta_{J}-$ shown on the vertical axis. The plots of $\mathbf{T}$ show the conditional probability of observing $\Delta_{J}-$ shown on the vertical axis, conditioned by $\Delta_{I}-$ shown on the horizontal axis. The presentation of accelerations and decelerations is restricted to events smaller than $80 \mathrm{~ms}$. However, such an interval of increments covers the crucial aspects of the heart beat dynamics.

The structure of the plots $\mathbf{A}$ and $\mathbf{T}$ is complex. Nevertheless, the gradient plots shown in the second line of Figures $\mathbf{1}$ and $\mathbf{2}$ reveal the basic geometrical properties of this structural complexity. Namely, we can observe how the probability changes under the modification of the arguments. The heads of the arrows indicate the direction in which the decrease is the strongest. The arrows' lengths correspond to the magnitude of this decrease.

In the last rows of Figures 1 and 2, we show graphs of the networks resulting from the signals studied, obtained by Pajek software (Batagelj and Mrvar, 2002). When presenting the networks in their graph form, we applied a special binning which allowed us to represent the crucial effects of aging in the clearest way. In the case of Figure 1, the RR-interval signals were binned at $48 \mathrm{~ms}$. As a consequence, the loop at the vertex 0 of the matrix A approximates the probability that two subsequent RR-increments were both smaller than $48 \mathrm{~ms}$. The growth of the width of these loops with age for the groups of older people points to the increasing presence of small changes in the dynamics (we add numbers to these loops to underline this observation). Together with this, the number of transitions greater than $48 \mathrm{~ms}$ is reduced.

The size $48 \mathrm{~ms}$ of a deceleration or an acceleration is close to the $50 \mathrm{~ms}$ value, which is the base of the popular index of vagal activity pNN50, describing the ratio of changes larger than $50 \mathrm{~ms}$ in a signal (TaskForce, 1996). Therefore, in the last row of Figure 2, we show parts of $\mathbf{T}$ networks describing the transition probability conditioned by an acceleration $-48 \mathrm{~ms}$ and a deceleration $+48 \mathrm{~ms}$. For the graphs of these networks, we used binning of RRintervals equal to $16 \mathrm{~ms}$. The conditional probabilities for other transitions obtained with this binning are presented in Table 1. The most probable transitions which are conditioned by a $\pm 48 \mathrm{~ms}$ change are shown in bold. 


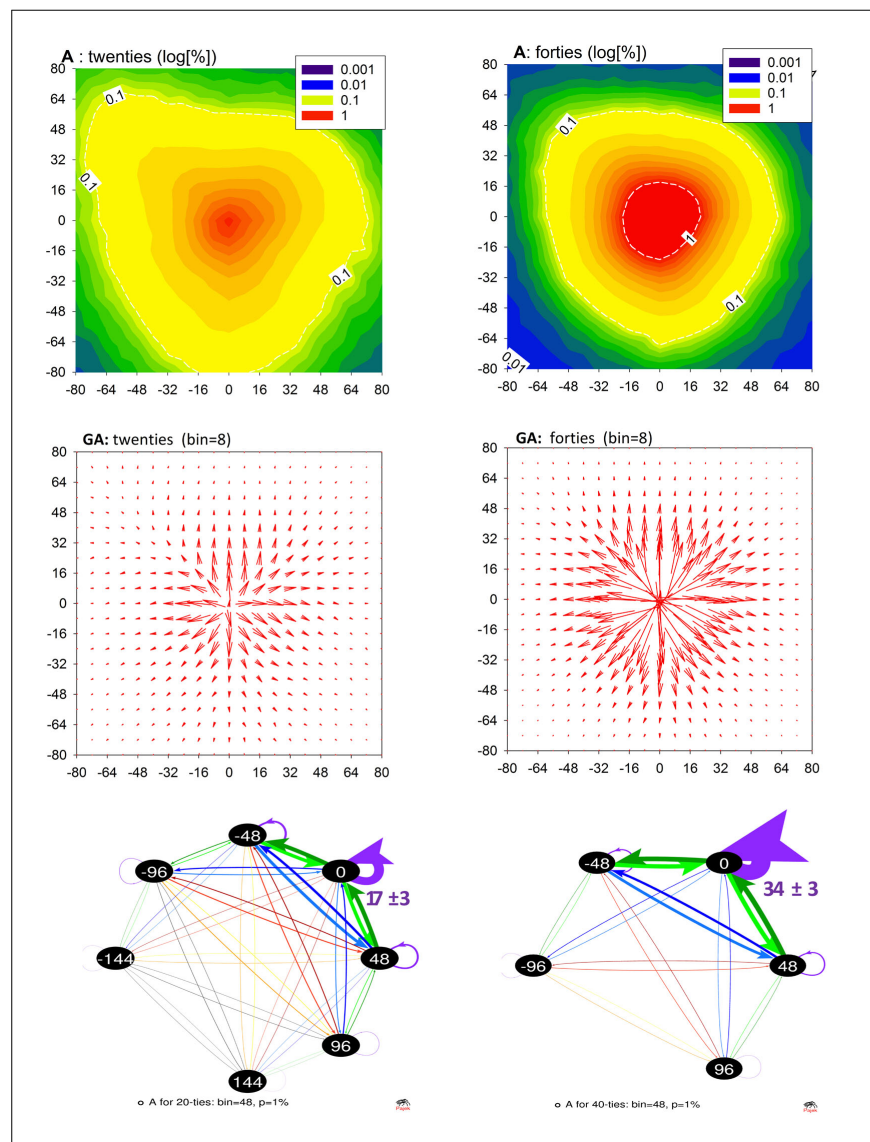

FIGURE 1 | Networks of transitions for aging population. Top row: matrices A averaged from matrices obtained from RR-signals pooled according to the age groups. Middle row: gradient matrices GA calculated from mean A. Bottom row: networks resulting from matrices $\mathbf{A}$ when RR-signals were binned
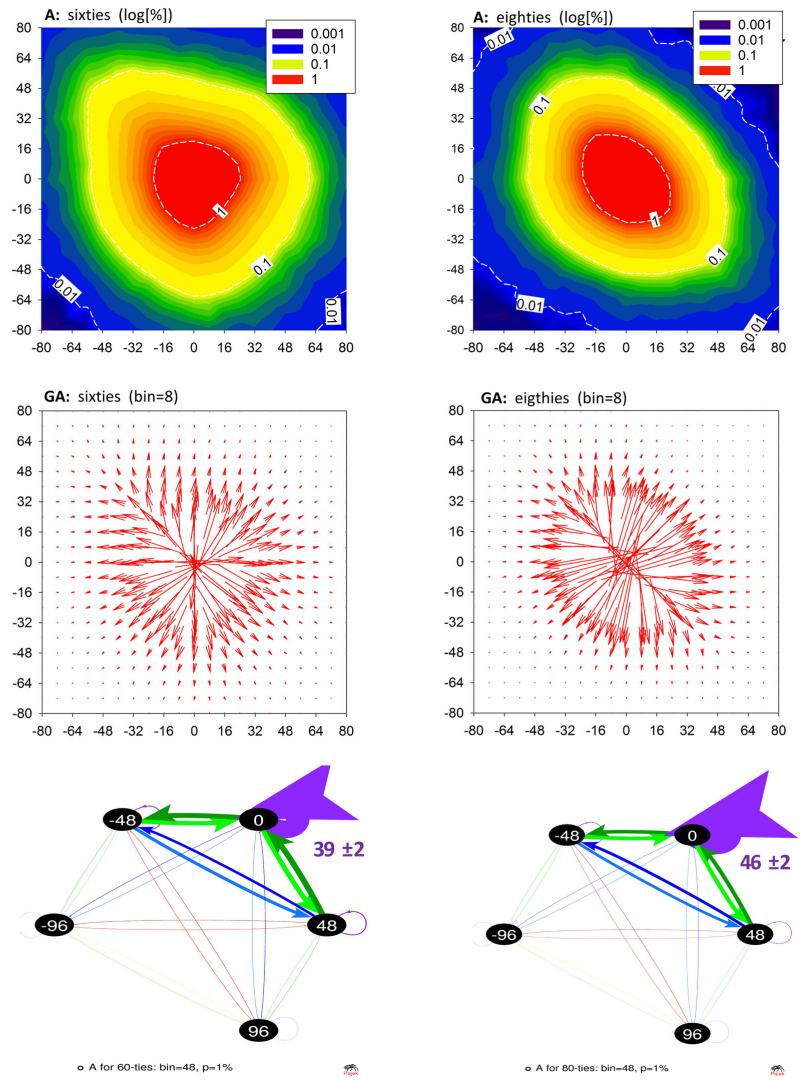

with bin $=48 \mathrm{~ms}$. Transitions are shown only between vertices of which the probability of occurrence is $>1 \%$. The growing role of small increments, as age progresses, is illustrated by providing numbers for the no-change events. Network graphs are visualized by Pajek software (Batagelj and Mrvar, 2002).
Finally, in Figure 3, properties of networks $\mathbf{A}$ and $\mathbf{T}$ are summarized in plots describing the decay of the deceleration capacity and entropy rate when the age of the group increases.

\section{Discussion}

The arrangement of the contour lines labeled $1 \%$ in the contour plots in Figure 1 (which represent pairs of events $\left(\delta \mathrm{RR}_{i}, \delta \mathrm{RR}_{i+1}\right)$ occurring with $1 \%$ probability) and lines labeled 0.06 in the contour plots in Figure 2 (describing the probability of a transition $\delta R R_{i+1}$ given that a transition $\delta R R_{i}$ has occurred), together with values collected in Table 1, reveal the dominant change in the heart beat dynamics caused by aging. Namely, the variability of possible $\left(\delta R R_{i}, \delta R R_{i+1}\right)$ events becomes gradually reduced with aging, which is further evidenced by the following mechanisms.

First, the reduction in the dynamics implies that the dynamics becomes increasingly dependent on the small $\delta R R$, namely it involves RR-increments of a size smaller than approximately $32 \mathrm{~ms}$. Network graphs in Figure 1, bottom row, constructed for the RR-signals binned with $48 \mathrm{~ms}$, extract parts of the dynamics focused on such small RR-increments and represent them together as a loop $(0,0)$. We observe that the regime dominated by the small change dynamics covers from about $17 \pm 3 \%$ at the age of 20 , through $34 \pm 3 \%$ at the age of 40 , and $39 \pm 2 \%$ at the age of 60 , to $46 \pm 2 \%$ at the age of 80 . The substantial increase in the lengths of the arrows in the gradient plots in Figure 1, middle row, is also a manifestation of this observation.

Second, there is an important qualitative transition in the probability of observing a pair $\left(\delta \mathrm{RR}_{i}, \delta \mathrm{RR}_{i+1}\right)$ when any large $\delta R R$ is encountered, i.e., an acceleration or a deceleration of a size greater than about $40 \mathrm{~ms}$ happens. The network graphs in Figure 2, bottom row, together with the values in Table 1, exemplify these changes as follows.

- 20s: both events, a large acceleration and a large deceleration, are slightly more likely to be followed by a deceleration of a size not greater than the preceding RR-increment. Specifically, according to the values presented in Table 1, we observe that:

- after acceleration $\delta R R_{i}=-48 \mathrm{~ms}$, the probability of seeing a deceleration of a size not greater than $\delta R R_{i}=48$, namely $P\left(16 \leq \delta R R_{i+1} \leq 48\right)$ is $31 \pm 2.9 \%$, and the probability of seeing an acceleration of a similar size, $P\left(-48 \leq \delta R R_{i+1} \leq-16\right)$ is $24 \pm 2.0 \%$;

- after deceleration $\delta R R_{i}=48 \mathrm{~ms}$, the probability of seeing a deceleration of a size not greater than $\delta R R_{i}=48, \quad P\left(16 \leq \delta R R_{i+1} \leq 48\right)$ is $31 \pm 2.9 \%$, while the 

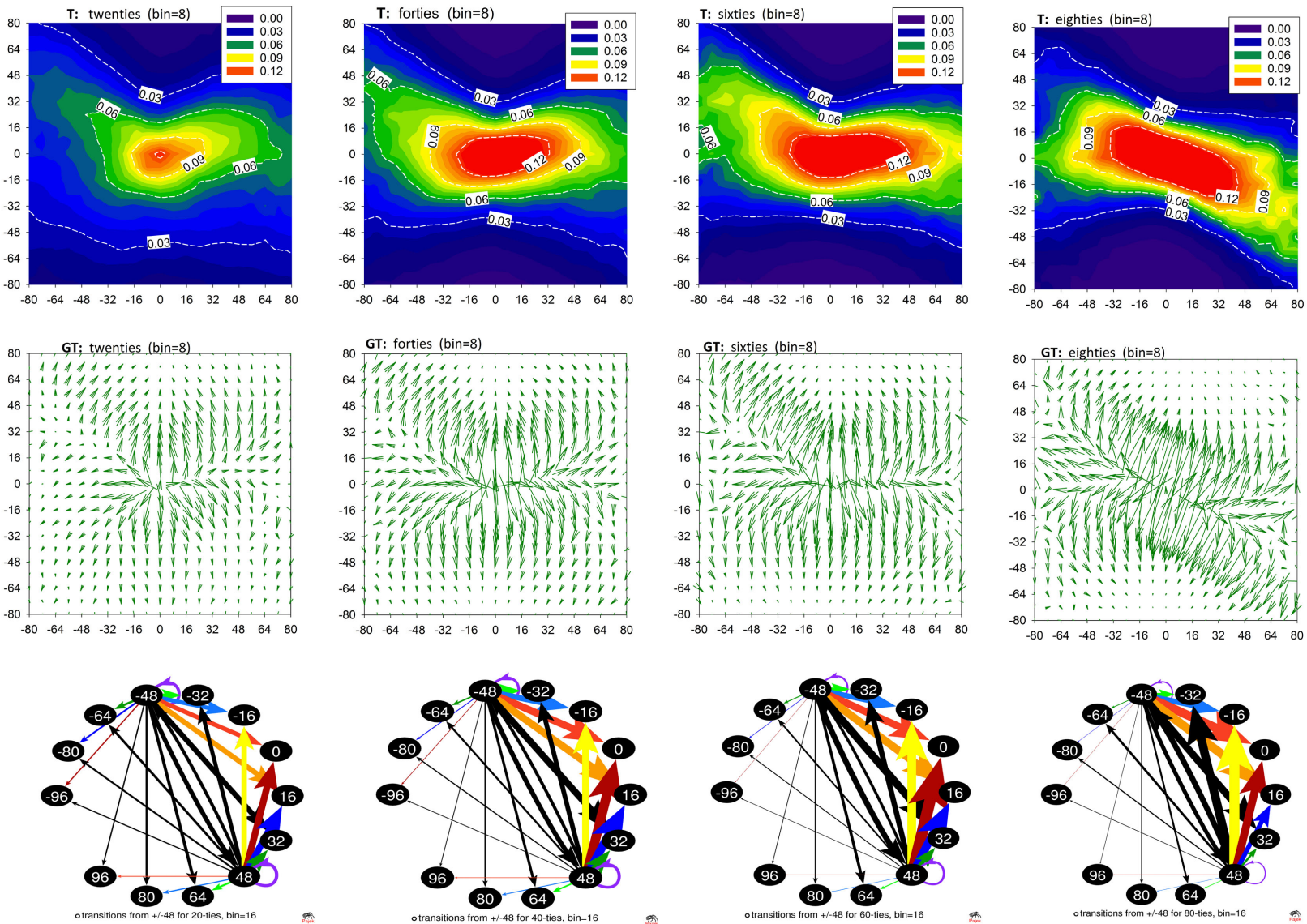

FIGURE 2 | Transition matrices in aging population. Top row: matrices $T$ averaged from matrices obtained from RR-signals pooled according to the age groups. Middle row: gradient matrices GT calculated from mean $\mathbf{T}$. Bottom row: networks resulting from matrices

probability of seeing an acceleration of a similar size $P\left(-48 \leq \delta R R_{i+1} \leq-16\right)$ is $26 \pm 2.0 \%$.

- 40s: despite a noticeable reduction in the overall variability, there is a strong increase in the probability of observing a large deceleration after a large acceleration or large deceleration. Using the description provided for the case of the $20 \mathrm{~s}$, we have:

- after $\delta R R_{i}=-48 \mathrm{~ms}: P\left(16 \leq \delta R R_{i+1} \leq 48\right)=40 \pm 4.5 \%$ and $P\left(-48 \leq \delta R R_{i+1} \leq-16\right)=27 \pm 2.2 \%$,

- after $\delta R R_{i}=48 \mathrm{~ms}: P\left(16 \leq \delta R R_{i+1} \leq 48\right)=34 \pm 3.5 \%$ and $P\left(-48 \leq \delta R R_{i+1} \leq-16\right)=29 \pm 3.3 \%$.

- 60s: after a large deceleration, although there is still a large probability of seeing a deceleration, there is also a large probability of seeing an acceleration. In particular,

- after $\delta R R_{i}=-48 \mathrm{~ms}: P\left(16 \leq \delta R R_{i+1} \leq 48\right)=48 \pm 6.5 \%$ and $P\left(-48 \leq \delta R R_{i+1} \leq-16\right)=23 \pm 3.3 \%$,

- after $\delta R R_{i}=48 \mathrm{~ms}: P\left(16 \leq \delta R R_{i+1} \leq 48\right)=41 \pm 4.8 \%$ and $P\left(-48 \leq \delta R R_{i+1} \leq-16\right)=35 \pm 4.2 \%$.

- 80s: large accelerations and decelerations are likely to occur alternately, which causes RR-intervals to change around the mean value in a pendulum-type motion rather than as a stochastic walk:

- after $\delta R R_{i}=-48 \mathrm{~ms}: P\left(16 \leq \delta R R_{i+1} \leq 48\right)=45 \pm 6.9 \%$ and $P\left(-48 \leq \delta R R_{i+1} \leq-16\right)=23 \pm 3.1 \%$,

- after $\delta R R_{i}=48 \mathrm{~ms}: P\left(16 \leq \delta R R_{i+1} \leq 48\right)=15 \pm 4.2 \%$ and $P\left(-48 \leq \delta R R_{i+1} \leq-16\right)=53 \pm 8.5 \%$.

The changes described above can be compared to the transition from equilibrium-like fluctuations in subjects in their forties to pendulum-type dynamical responses in subjects in their eighties. The gradient plots GA demonstrate additionally the increase of the steepness of the distributions $\mathbf{A}$ around the no-change event, and the differences in the symmetry of the events which occur in the elderly.

Since the occurrence of large RR-increments could be related to either strong vagal activation or to vagal withdrawal combined with sympathetic stimulation (Martini et al., 2011; Poirier, 2014), we can considered our results to reveal diminishing vagal activity and/or diminishing cooperation between the vagal and the sympathetic part of the ANS with aging. This observation is supported directly by a strong decay in the deceleration capacity; see Figure 3 left. 
TABLE 1 | Core parts of T: $P\left(\Delta_{J} \mid \Delta_{l}\right)$ in the case of bin $=\mathbf{1 6} \mathrm{ms}$ for different age groups are given as the group mean \pm SEM

\begin{tabular}{|c|c|c|c|c|c|c|c|c|c|c|c|}
\hline $\begin{array}{l}\Delta_{J} \rightarrow \\
\Delta_{I \downarrow}\end{array}$ & -80 & -64 & -48 & -32 & -16 & 0 & 16 & 32 & 48 & 64 & 80 \\
\hline \multicolumn{12}{|c|}{ TWENTIES } \\
\hline-80 & $3 \pm 0.4$ & $4 \pm 0.5$ & $5 \pm 0.5$ & $6 \pm 0.6$ & $7 \pm 0.6$ & $8 \pm 0.7$ & $9 \pm 0.7$ & $10 \pm 0.9$ & $9 \pm 1.0$ & $8 \pm 1.0$ & $6 \pm 0.9$ \\
\hline-64 & $3 \pm 0.4$ & $4 \pm 0.5$ & $6 \pm 0.5$ & $7 \pm 0.5$ & $8 \pm 0.6$ & $9 \pm 0.7$ & $10 \pm 0.8$ & $11 \pm 1.0$ & $10 \pm 1.0$ & $8 \pm 0.9$ & $5 \pm 0.8$ \\
\hline-48 & $4 \pm 0.4$ & $5 \pm 0.4$ & $6 \pm 0.5$ & $8 \pm 0.6$ & $10 \pm 0.7$ & $10 \pm 0.7$ & $11 \pm 1.0$ & $11 \pm 1.0$ & $9 \pm 0.9$ & $6 \pm 0.6$ & $4 \pm 0.5$ \\
\hline-32 & $4 \pm 0.4$ & $5 \pm 0.4$ & $7 \pm 0.5$ & $9 \pm 0.7$ & $11 \pm 1.0$ & $13 \pm 1.2$ & $13 \pm 1.4$ & $11 \pm 1.0$ & $7 \pm 0.6$ & $5 \pm 0.4$ & $3 \pm 0.3$ \\
\hline-16 & $3 \pm 0.3$ & $5 \pm 0.4$ & $7 \pm 0.6$ & $9 \pm 0.8$ & $14 \pm 1.6$ & $18 \pm 2.8$ & $15 \pm 2.4$ & $9 \pm 0.9$ & $5 \pm 0.3$ & $3 \pm 0.2$ & $2 \pm 0.2$ \\
\hline 0 & $3 \pm 0.3$ & $5 \pm 0.4$ & $7 \pm 0.6$ & $10 \pm 1.0$ & $17 \pm 2.7$ & $22 \pm 3.8$ & $14 \pm 2.1$ & $7 \pm 0.6$ & $4 \pm 0.3$ & $2 \pm 0.1$ & $1 \pm 0.1$ \\
\hline 16 & $3 \pm 0.3$ & $5 \pm 0.4$ & $7 \pm 0.6$ & $10 \pm 0.9$ & $15 \pm 1.9$ & $19 \pm 3.0$ & $15 \pm 1.8$ & $9 \pm 0.8$ & $5 \pm 0.3$ & $3 \pm 0.2$ & $2 \pm 0.1$ \\
\hline 32 & $4 \pm 0.3$ & $5 \pm 0.5$ & $7 \pm 0.6$ & $9 \pm 0.7$ & $12 \pm 1.0$ & $16 \pm 1.6$ & $15 \pm 1.6$ & $10 \pm 1.0$ & $6 \pm 0.5$ & $3 \pm 0.3$ & $2 \pm 0.2$ \\
\hline 48 & $4 \pm 0.4$ & $5 \pm 0.5$ & $7 \pm 0.6$ & $8 \pm 0.7$ & $11 \pm 0.7$ & $14 \pm 1.1$ & $14 \pm 1.4$ & $10 \pm 0.9$ & $7 \pm 0.6$ & $4 \pm 0.3$ & $3 \pm 0.3$ \\
\hline 64 & $4 \pm 0.5$ & $5 \pm 0.6$ & $7 \pm 0.7$ & $9 \pm 0.9$ & $10 \pm 0.9$ & $13 \pm 1.1$ & $12 \pm 1.0$ & $9 \pm 0.8$ & $7 \pm 0.6$ & $4 \pm 0.4$ & $3 \pm 0.3$ \\
\hline 80 & $4 \pm 0.5$ & $6 \pm 0.7$ & $7 \pm 0.9$ & $9 \pm 1.0$ & $11 \pm 1.2$ & $11 \pm 1.2$ & $10 \pm 0.9$ & $8 \pm 0.7$ & $6 \pm 0.5$ & $4 \pm 0.4$ & $3 \pm 0.3$ \\
\hline \multicolumn{12}{|c|}{ FORTIES } \\
\hline-80 & $2 \pm 0.7$ & $3 \pm 0.7$ & $5 \pm 0.8$ & $6 \pm 0.8$ & $8 \pm 1.0$ & $10 \pm 1.4$ & $11 \pm 2.0$ & $12 \pm 2.5$ & $13 \pm 3.1$ & $10 \pm 2.7$ & $7 \pm 2.0$ \\
\hline-64 & $2 \pm 0.5$ & $3 \pm 0.6$ & $5 \pm 0.6$ & $7 \pm 0.8$ & $10 \pm 0.9$ & $12 \pm 1.2$ & $13 \pm 1.6$ & $13 \pm 2.1$ & $12 \pm 2.2$ & $8 \pm 1.9$ & $5 \pm 1.2$ \\
\hline-48 & $2 \pm 0.4$ & $3 \pm 0.5$ & $5 \pm 0.6$ & $9 \pm 0.7$ & $13 \pm 0.9$ & $16 \pm 1.1$ & $16 \pm 1.3$ & $14 \pm 1.7$ & $10 \pm 1.5$ & $5 \pm 1.0$ & $3 \pm 0.6$ \\
\hline-32 & $1 \pm 0.3$ & $3 \pm 0.3$ & $5 \pm 0.4$ & $10 \pm 0.7$ & $17 \pm 1.2$ & $21 \pm 1.3$ & $18 \pm 1.2$ & $13 \pm 1.0$ & $6 \pm 0.7$ & $3 \pm 0.4$ & $1 \pm 0.2$ \\
\hline-16 & $1 \pm 0.2$ & $2 \pm 0.3$ & $4 \pm 0.4$ & $9 \pm 0.6$ & $20 \pm 1.5$ & $27 \pm 2.6$ & $19 \pm 1.6$ & $9 \pm 0.6$ & $4 \pm 0.3$ & $1 \pm 0.2$ & $1 \pm 0.1$ \\
\hline 0 & $1 \pm 0.2$ & $2 \pm 0.3$ & $4 \pm 0.4$ & $9 \pm 0.6$ & $21 \pm 1.9$ & $32 \pm 4.2$ & $18 \pm 1.6$ & $7 \pm 0.4$ & $2 \pm 0.2$ & $1 \pm 0.1$ & $0 \pm 0.1$ \\
\hline 16 & $1 \pm 0.2$ & $2 \pm 0.3$ & $5 \pm 0.4$ & $10 \pm 0.6$ & $20 \pm 1.4$ & $28 \pm 2.4$ & $19 \pm 1.4$ & $8 \pm 0.5$ & $3 \pm 0.2$ & $1 \pm 0.1$ & $1 \pm 0.1$ \\
\hline 32 & $2 \pm 0.4$ & $3 \pm 0.5$ & $5 \pm 0.6$ & $9 \pm 0.7$ & $16 \pm 0.9$ & $23 \pm 1.6$ & $19 \pm 1.5$ & $10 \pm 0.6$ & $4 \pm 0.4$ & $2 \pm 0.2$ & $1 \pm 0.1$ \\
\hline 48 & $2 \pm 0.6$ & $4 \pm 0.7$ & $6 \pm 1.0$ & $9 \pm 1.1$ & $14 \pm 1.2$ & $19 \pm 1.6$ & $17 \pm 1.8$ & $11 \pm 1.0$ & $6 \pm 0.7$ & $3 \pm 0.4$ & $2 \pm 0.3$ \\
\hline 64 & $3 \pm 0.7$ & $5 \pm 1.0$ & $7 \pm 1.2$ & $10 \pm 1.5$ & $12 \pm 1.7$ & $16 \pm 2.1$ & $14 \pm 2.0$ & $11 \pm 1.6$ & $7 \pm 1.2$ & $4 \pm 0.8$ & $2 \pm 0.5$ \\
\hline 80 & $4 \pm 0.9$ & $5 \pm 1.1$ & $7 \pm 1.5$ & $9 \pm 1.8$ & $11 \pm 2.0$ & $13 \pm 2.4$ & $12 \pm 2.3$ & $10 \pm 2.1$ & $7 \pm 1.4$ & $5 \pm 1.1$ & $3 \pm 0.7$ \\
\hline \multicolumn{12}{|c|}{ SIXTIES } \\
\hline-80 & $1 \pm 0.5$ & $3 \pm 1.0$ & $5 \pm 1.7$ & $7 \pm 2.0$ & $9 \pm 2.2$ & $11 \pm 2.5$ & $12 \pm 3.2$ & $13 \pm 3.5$ & $13 \pm 3.4$ & $10 \pm 3.1$ & $7 \pm 2.0$ \\
\hline-64 & $1 \pm 0.4$ & $2 \pm 0.7$ & $4 \pm 1.0$ & $7 \pm 1.5$ & $10 \pm 1.7$ & $13 \pm 2.2$ & $14 \pm 2.7$ & $16 \pm 3.1$ & $13 \pm 3.1$ & $9 \pm 2.5$ & $5 \pm 1.5$ \\
\hline-48 & $1 \pm 0.3$ & $2 \pm 0.5$ & $4 \pm 0.8$ & $7 \pm 1.1$ & $12 \pm 1.4$ & $16 \pm 1.3$ & $17 \pm 1.6$ & $17 \pm 2.4$ & $12 \pm 2.3$ & $6 \pm 1.3$ & $3 \pm 0.7$ \\
\hline-32 & $1 \pm 0.2$ & $2 \pm 0.4$ & $3 \pm 0.5$ & $8 \pm 0.8$ & $15 \pm 1.1$ & $22 \pm 1.3$ & $21 \pm 1.4$ & $16 \pm 1.7$ & $7 \pm 1.0$ & $3 \pm 0.5$ & $1 \pm 0.3$ \\
\hline-16 & $1 \pm 0.2$ & $1 \pm 0.3$ & $3 \pm 0.4$ & $9 \pm 0.6$ & $19 \pm 1.2$ & $28 \pm 2.6$ & $22 \pm 1.7$ & $11 \pm 0.7$ & $4 \pm 0.3$ & $1 \pm 0.2$ & $1 \pm 0.1$ \\
\hline 0 & $1 \pm 0.1$ & $1 \pm 0.3$ & $3 \pm 0.4$ & $10 \pm 0.6$ & $22 \pm 1.9$ & $33 \pm 4.4$ & $19 \pm 1.7$ & $7 \pm 0.4$ & $2 \pm 0.2$ & $1 \pm 0.1$ & $0 \pm 0.1$ \\
\hline 16 & $1 \pm 0.2$ & $2 \pm 0.3$ & $4 \pm 0.5$ & $11 \pm 0.7$ & $22 \pm 1.5$ & $31 \pm 2.6$ & $18 \pm 1.2$ & $7 \pm 0.5$ & $2 \pm 0.2$ & $1 \pm 0.1$ & $0 \pm 0.1$ \\
\hline 32 & $1 \pm 0.3$ & $2 \pm 0.5$ & $5 \pm 0.8$ & $11 \pm 1.2$ & $20 \pm 1.3$ & $27 \pm 1.9$ & $19 \pm 1.4$ & $8 \pm 0.8$ & $3 \pm 0.5$ & $1 \pm 0.2$ & $1 \pm 0.1$ \\
\hline 48 & $2 \pm 0.5$ & $3 \pm 0.8$ & $6 \pm 1.0$ & $11 \pm 1.7$ & $18 \pm 1.9$ & $23 \pm 2.6$ & $17 \pm 2.1$ & $9 \pm 1.3$ & $5 \pm 0.8$ & $2 \pm 0.4$ & $1 \pm 0.2$ \\
\hline 64 & $2 \pm 0.8$ & $4 \pm 1.0$ & $7 \pm 1.4$ & $12 \pm 2.1$ & $17 \pm 2.8$ & $19 \pm 3.5$ & $15 \pm 3.3$ & $9 \pm 1.9$ & $5 \pm 1.2$ & $3 \pm 0.8$ & $1 \pm 0.5$ \\
\hline 80 & $4 \pm 1.2$ & $5 \pm 1.4$ & $8 \pm 2.0$ & $13 \pm 2.9$ & $14 \pm 3.2$ & $16 \pm 4.3$ & $14 \pm 3.8$ & $8 \pm 1.9$ & $6 \pm 1.6$ & $3 \pm 1.0$ & $2 \pm 0.5$ \\
\hline \multicolumn{12}{|c|}{ EIGHTIES } \\
\hline-80 & $2 \pm 1.1$ & $3 \pm 1.5$ & $4 \pm 2.3$ & $8 \pm 2.7$ & $10 \pm 3.1$ & $12 \pm 3.5$ & $11 \pm 2.8$ & $9 \pm 2.4$ & $9 \pm 2.4$ & $6 \pm 1.8$ & $3 \pm 1.1$ \\
\hline-64 & $1 \pm 0.8$ & $2 \pm 1.0$ & $4 \pm 1.8$ & $7 \pm 2.3$ & $11 \pm 2.9$ & $16 \pm 3.8$ & $14 \pm 3.1$ & $13 \pm 3.1$ & $10 \pm 2.3$ & $6 \pm 1.2$ & $3 \pm 0.8$ \\
\hline-48 & $1 \pm 0.3$ & $2 \pm 0.7$ & $3 \pm 0.9$ & $7 \pm 1.7$ & $13 \pm 2.5$ & $20 \pm 3.5$ & $20 \pm 2.9$ & $16 \pm 2.4$ & $9 \pm 1.6$ & $4 \pm 0.8$ & $2 \pm 0.5$ \\
\hline-32 & $0 \pm 0.1$ & $1 \pm 0.3$ & $2 \pm 0.5$ & $5 \pm 0.9$ & $14 \pm 1.7$ & $24 \pm 1.9$ & $26 \pm 1.7$ & $16 \pm 1.6$ & $6 \pm 0.9$ & $2 \pm 0.4$ & $1 \pm 0.2$ \\
\hline-16 & $0 \pm 0.1$ & $0 \pm 0.1$ & $1 \pm 0.2$ & $5 \pm 0.5$ & $16 \pm 1.1$ & $33 \pm 2.7$ & $29 \pm 2.5$ & $11 \pm 0.7$ & $3 \pm 0.4$ & $1 \pm 0.2$ & $0 \pm 0.1$ \\
\hline 0 & $0 \pm 0.1$ & $0 \pm 0.1$ & $1 \pm 0.2$ & $6 \pm 0.5$ & $22 \pm 1.7$ & $40 \pm 4.7$ & $21 \pm 1.7$ & $6 \pm 0.4$ & $2 \pm 0.3$ & $1 \pm 0.1$ & $0 \pm 0.0$ \\
\hline 16 & $0 \pm 0.1$ & $1 \pm 0.2$ & $3 \pm 0.4$ & $10 \pm 0.7$ & $30 \pm 2.7$ & $33 \pm 2.7$ & $15 \pm 1.3$ & $5 \pm 0.6$ & $1 \pm 0.3$ & $0 \pm 0.1$ & $0 \pm 0.1$ \\
\hline 32 & $1 \pm 0.3$ & $2 \pm 0.5$ & $6 \pm 0.9$ & $18 \pm 1.9$ & $28 \pm 1.8$ & $24 \pm 2.0$ & $12 \pm 1.6$ & $5 \pm 0.9$ & $2 \pm 0.5$ & $1 \pm 0.3$ & $0 \pm 0.2$ \\
\hline 48 & $2 \pm 0.7$ & $5 \pm 1.2$ & $12 \pm 2.6$ & $19 \pm 2.9$ & $22 \pm 3.0$ & $17 \pm 2.8$ & $10 \pm 2.1$ & $5 \pm 1.3$ & $2 \pm 0.8$ & $1 \pm 0.5$ & $1 \pm 0.4$ \\
\hline 64 & $5 \pm 1.1$ & $10 \pm 2.2$ & $15 \pm 3.3$ & $16 \pm 3.1$ & $15 \pm 2.7$ & $13 \pm 2.8$ & $8 \pm 2.3$ & $4 \pm 1.8$ & $2 \pm 1.3$ & $2 \pm 0.9$ & $1 \pm 0.6$ \\
\hline 80 & $8 \pm 1.9$ & $13 \pm 3.1$ & $13 \pm 3.0$ & $14 \pm 3.3$ & $11 \pm 2.7$ & $10 \pm 2.8$ & $7 \pm 3.1$ & $4 \pm 2.0$ & $3 \pm 1.5$ & $2 \pm 1.3$ & $1 \pm 0.8$ \\
\hline
\end{tabular}

$\Delta_{j}$ 's are given in columns for $\Delta_{j}$ 's described in rows. The bold fonts are used to lead the eye to the most probable transitions, occurring after large accelerations $\Delta_{l}=-48$ ms and larger decelerations $\Delta_{l}=48 \mathrm{~ms}$, which are distinct from no-change events. These numbers result in the clock-like picture in Figure 4.

The mean transition matrices shown in Figure 2 illustrate the short-term dependence between the subsequent heart beats. We see that the core probability, which involves small variations around the no-change event at the age of 20 , spreads to events other than the no-change event when the subjects are in their forties. Moreover, the probability of the next $\delta R R(i+1)$ appears to be independent of the last $\delta R R(i)$ for changes from $-32 \mathrm{~ms}$ to $32 \mathrm{~ms}$. This independence spreads, but for the decelerations only, up to $64 \mathrm{~ms}$ in the case of subjects in their sixties. Accelerations are characterized by the probabilities dependent on the size of the preceding acceleration. The greater the acceleration, the larger the probability is of a deceleration in the next step. At the age of 80 , such alternations, which can be compared to pendulum dynamics, are also observed in the decelerations.

This pendulum-like dynamics is damped because the increment which follows a given event is smaller. Moreover, such gradual stabilization of the transition probabilities with age is evidenced by a decrease in the entropy rate, see Figure 3. Because of this effect, the location of the maxima in the transition probability depends on $\Delta_{J}$, which is clearly demonstrated in the gradient plots GT. The occurrence of the pendulum-like dynamics is represented in detail in Table 1, where the results obtained from signals 


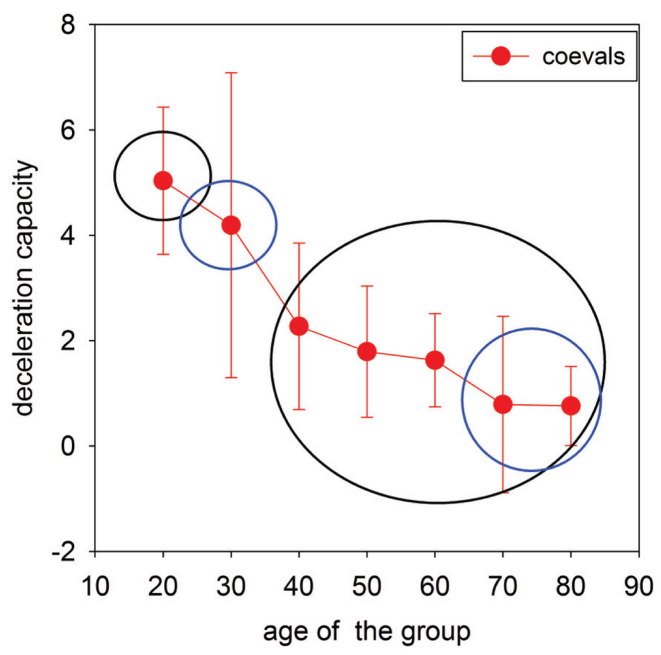

FIGURE 3 | Summary indices for network representations of age-induced alternations in heart rate dynamics. Left: Deceleration capacity (median \pm larger distance to the quartile) calculated for individual $\delta R R$ signals and then pooled in the age groups. Circles separate statistically different groups found by Kruskal-Wallis ANOVA on

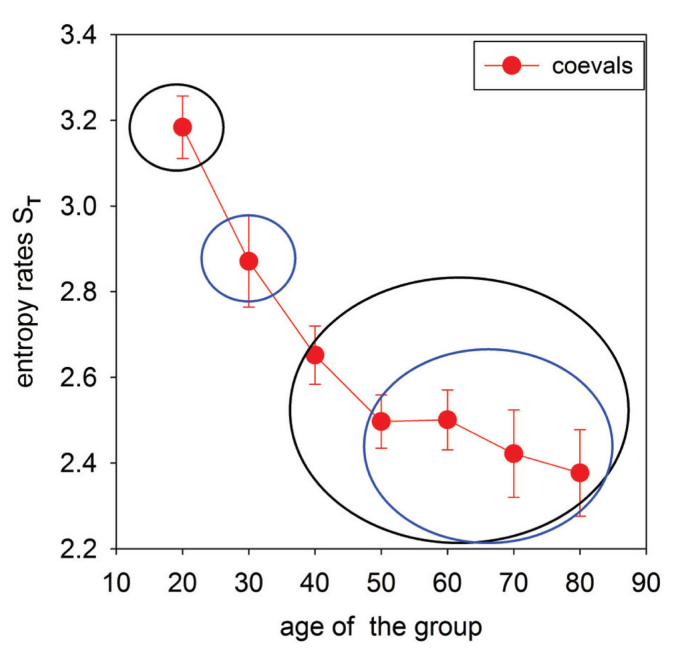

Ranks with the Dunn method used for comparisons between pairs. Right: Transition entropy (mean \pm SEM) calculated for $\delta R R$ signals pooled in age groups. Circles separate statistically different groups found by ANOVA with the Holm-Sidak method used for comparisons between pairs. binned with $\operatorname{bin}=16 \mathrm{~ms}$ are provided. From Table 1 we see that, although transitions to no-change events are the most probable in elderly humans, independently of the size of RR-increments, the pendulum oscillations are present.

RR-signals are known to have $1 / f$ scaling properties with the Hurst exponent gradually approaching $H=0.2$ with aging (Struzik et al., 2006) for all but the shortest timescales ( $>20$ heart beats). This phenomenon corresponds with increasingly antipersistent behavior of RR-signals at the smallest temporal resolutions with increasing age. Such simple antipersistent dynamics is also characteristic of the heart rhythm of patients after heart transplantation, that is, for subjects with denervated hearts (Makowiec et al., 2014). Table 1 supports the conclusion that the antipersistent features are attributed to accelerations independently of the subject's age, while the decelerations change from a slight persistency to antipersistency with the subject's age. We can picture the changes by drawing lines in GT plots, which connect maxima of transition probabilities, see Figure 4. These lines metaphorically resemble the hands of a clock. We can indeed read the passing of time in our "chronograph" of heart rate dynamics: 10 min past ten for subjects in their twenties, a quarter past ten for subjects in their sixties, and 20 past ten for subjects in their eighties. The relative angle between the arrows - the hands of the metaphoric chronograph - reflects the degree of antipersistency; however, the absolute position of the arrows, the ridges of the maxima of the gradient, is also of physiological relevance.

The increase in pendulum-like oscillatory antipersistency may involve the very mechanism responsible for the decrease in entropy with age, also observed previously (Viola et al., 2011; Makowiec et al., 2015b). It is as of yet unclear whether solely ANS neural factors are behind the physiological origins of this mechanism. It may be related to feedback compensatory dynamics due to the decrease in baroreflex sensitivity with age. Possibly, the gradual stiffening of the vasculature and hypertrophy of the cardiac muscle with age plays a role in the genesis of this effect. Both these factors may be present and may convolve with the altering spectrum of autonomic control. The progressive impairment of the autonomic balance with age (Crasset et al., 2001; Brandenberger et al., 2003) does not resolve the question of the direction of the causal relationship - whether the increase in the sympathetic dominance is responsible for the diminishing appearance of deep sleep or whether the lack of deep sleep in the elderly leads to lesser evidence of the vagal tone. The debate remains open, as some authors argue (Schmitt et al., 2009) that the structure of sleep dominates over aging processes and "overrides" the effects of aging on HRV. This could potentially explain the fact of decreased vagal presence despite the progressive loss of sympathetic neurons with age (Struzik et al., 2006).

Sleep can in general be assumed to be a period of human activity which is free of external stimulation. Therefore, the nocturnal part of a 24-h Holter recording provides a good possibility of observing the state of the autonomic baseline (Stein and $\mathrm{Pu}, 2012$; Tobaldini et al., 2013; Chouchou and Desseilles, 2014). Indeed, a recent review (Stein and $\mathrm{Pu}, 2012$ ) advocates using nocturnal records for HRV analysis, while (Chouchou and Desseilles, 2014) reviews tantalizing possibilities of the quantitative assessment of not only autonomic but also higher nervous centers through analysis of sleep HRV records. However, as sleep is organized in cycles, each lasting about $90 \mathrm{~min}$, stages of slow wave sleep (non-rapid eye movement sleep: NREM) are followed by rapid eye movement sleep (REM) (Guyton and Hall, 2006). HRV has indeed been found to be strongly affected by the sleep organization - vagal modulation and sympathetic activity follow the sleep stages (Monti et al., 2002; Schumann et al., 2010). It is generally recognized that NREM is characterized by a predominant parasympathetic drive, while the REM stage exhibits increased sympathetic modulation and a loss of parasympathetic control. Also, the brain is more active in REM sleep. REM sleep and arousal 

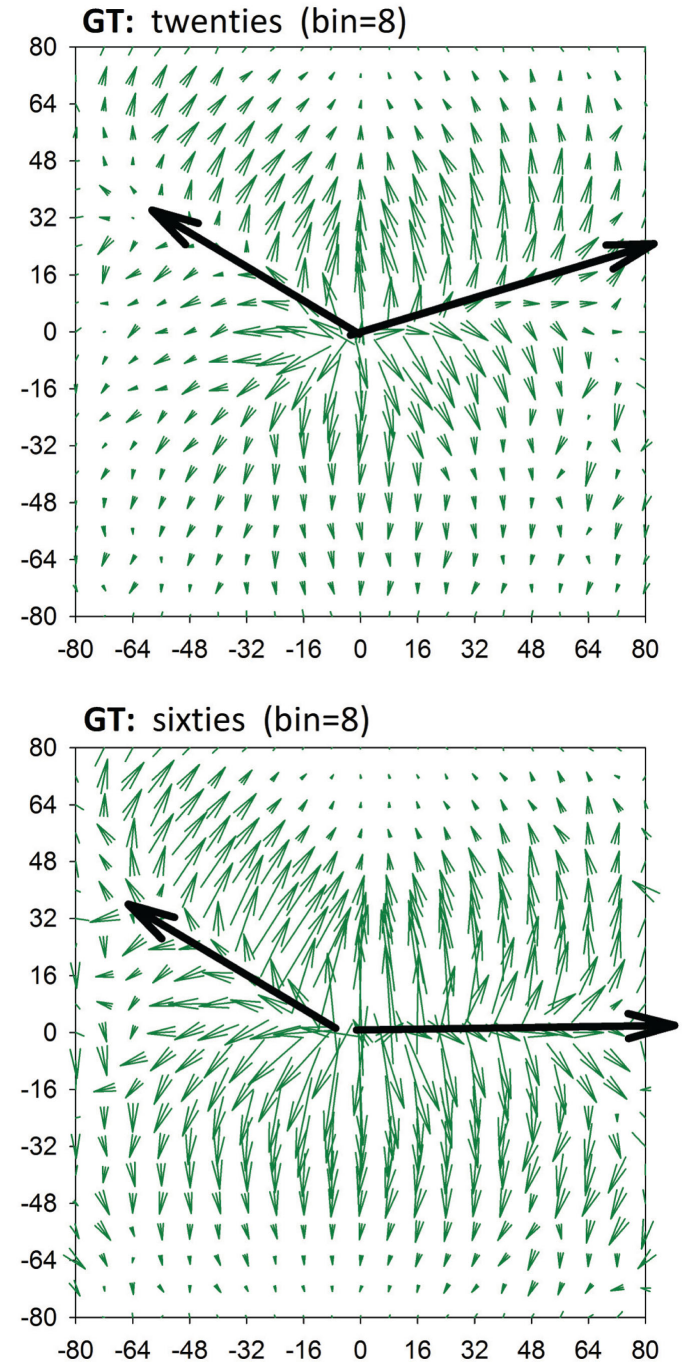

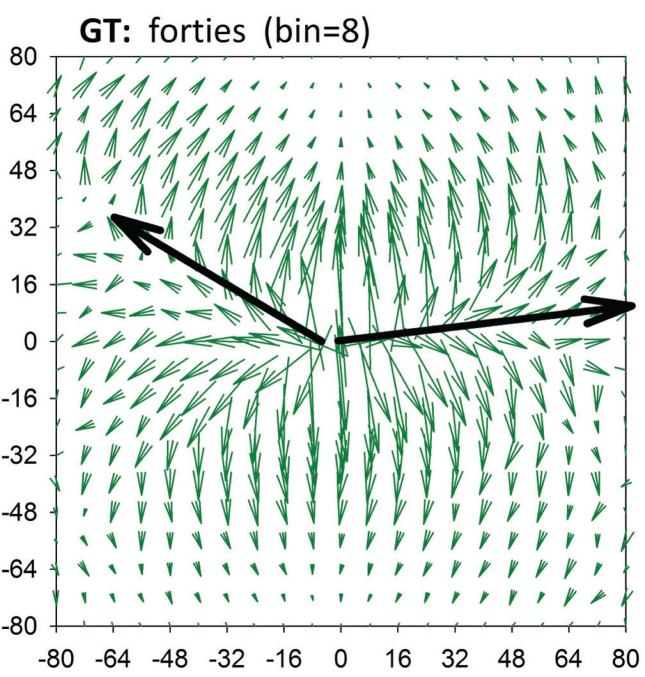

GT: eighties (bin=8)

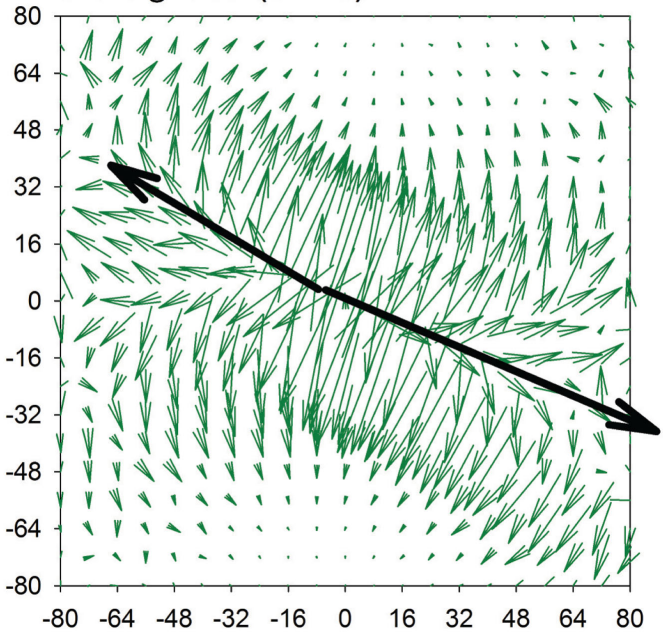

FIGURE 4 | A clock of autonomic aging made by linking points for which values of T(I,*) are maximal.

from sleep presumably reflect central autonomic commands leading to transient periods of tachycardia (Trinder, 2008). It has been found that these transitions lead to significantly higher values of SD of RR intervals for REM sleep than for deep NREM sleep, independently of age (Schmitt et al., 2009). However, the index of very short-term variability, the square root of the mean of the sum of the squares of differences (RMSSD) has been found to be insensitive to non-stationarities caused by bursts of sympathetic activity during different sleep stages independently of age (Schmitt et al., 2009). Since our investigations are related to short-term variability, we can suppose that our results are not strongly influenced by sleep stages or sleep transitions.

\section{Summary}

The complexity of the mechanisms involved in the phenomenon of heart rate variability is challenging, and the existing measures of this complexity are not satisfactory. The proposed method provides easily readable graphs revealing the complexity of the autonomic control, to date not assessed in comparable detail. The intricate topological structure of these graphs helps to reveal the effects of sympathetic and vagal modulation on heart rate dynamics. Additionally, the two measures proposed provide the means to summarize the graphs' structure: (i) the approximate deceleration capacity and (ii) the entropy rates.

Significant age-related changes in heart beat-to-beat dynamics have been observed using the geometrical tools of the method introduced: from the large variety of possible movements at the age of 20 , to the strong antipersistency similar to pendulum dynamics, which becomes dominant in subjects in their eighties.

Aging and its effects on the entire cardiovascular system, and specifically on the autonomic regulation, are themselves complex processes and capturing this in HRV remains an open problem. In particular, causal, possibly non-linear interactions (Porta et al., 2014) may be involved. Our geometrically oriented methodology may contribute to the range of analytic methods and help in further elucidating complex mechanisms of aging. 


\section{Author Contributions}

DW, AK, MZB: the acquisition of data for the work; DM: drafting of the work; DM, ZRS: critically revising the significant intellectual content of the work, and final approval of the published version.

\section{References}

Batagelj, V., and Mrvar, A. (2002). "Pajek analysis and visualization of large networks," in Graph Drawing, Volume 2265 of Lecture Notes in Computer Science, eds P. Mutzel, M. Jünger, and S. Leipert (Berlin: Springer), 477-478.

Beckers, F., Verheyden, B., and Aubert, A. E. (2006). Aging and nonlinear heart rate control in a healthy population. Am. J. Physiol. Heart Circ. Physiol. 290, H2560-H2570. doi:10.1152/ajpheart.00903.2005

Brandenberger, G., Viola, A. U., Ehrhart, J., Charloux, A., Geny, B., Piquard, F., et al. (2003). Age-related changes in cardiac autonomic control during sleep. J. Sleep Res. 12, 173-180. doi:10.1046/j.1365-2869.2003.00353.x

Callegaro, C. C., and Taylor, J. (2010). Age-related effects of vagotonic atropine on cardiovagal baroreflex gain. Neurobiol. Aging 33, 368-374. doi:10.1016/j. neurobiolaging.2010.03.018

Campanharo, A. S. L. O., Sirer, M. I., Malmgren, R. D., Ramos, F. M., and Amaral, L. A. N. (2011). Duality between time series and networks. PLoS ONE 6:e23378. doi:10.1371/journal.pone.0023378

Chouchou, F., and Desseilles, M. (2014). Heart rate variability: a tool to explore the sleeping brain? Front. Neurosci. 8:402. doi:10.3389/fnins.2014.00402

Crasset, V., Mezzetti, S., Antoine, M., Linkowski, P., Degaute, J. P., and van de Borne, P. (2001). Effects of aging and cardiac denervation on heart rate variability during sleep. Circulation 103, 84-88. doi:10.1161/01.CIR.103.1.84

Donner, R. V., Zou, Y., Donges, J. F., Marwan, N., and Kurths, J. (2010). Recurrence networks - a novel paradigm for nonlinear time series analysis. New J. Phys. 12, 033025. doi:10.1088/1367-2630/12/3/033025

Epstein, L. J., Kristo, D., Strollo, P. J., Friedman, N., Malhotra, A., Patil, S. P., et al. (2009). Adult obstructive sleep apnea task force of the American academy of sleep medicine. Clinical guideline for the evaluation, management and longterm care of obstructive sleep apnea in adults. J. Clin. Sleep Med. 5, 263-276. doi:10.1161/01.CIR.93.5.1043

Esler, M. D., Thompson, J. M., Kaye, D. M., Turner, A. G., Jennings, G. L., Cox, H. S., et al. (1995). Effects of aging on the responsiveness of the human cardiac sympathetic nerves to stressors. Circulation 91, 351-358. doi:10.1161/01.CIR.91. 2.351

Goldberger, J. J., Cain, M. E., Hohnloser, S. H., Kadish, A. H., Knight, B. P., Lauer, M. S., et al. (2008). American Heart Association/American College of Cardiology Foundation/Heart Rhythm Society scientific statement on noninvasive risk stratification techniques for identifying patients at risk for sudden cardiac death: a scientific statement from the American Heart Association Council on Clinical Cardiology, Committee on Electrocardiography and Arrhythmias, and Council on Epidemiology and Prevention. Circulation 118, 1497-1518. doi:10. 1161/CIRCULATIONAHA.107.189375

Guyton, A. C., and Hall, J. E. (2006). Textbook of Medical Physiology. Philadelphia, PA: Elsevier Saunders Company.

Makowiec, D., Graff, B., Kaczkowska, A., Graff, G., Wejer, D., Wdowczyk, J., et al. (2015a). in Visualization of Short-Term Heart Period Variability with Network Tools as a Method for Quantifying Autonomic Drive, to appear in "Interpretation of ECG Time Series Using Inter-Beat Variability Analysis: From Engineering to Medicine", eds J. Herbert, K. Ahsan, and C. David (CRC Press, Taylor \& Francis Group).

Makowiec, D., Kaczkowska, A., Wejer, D., Żarczyńska-Buchowiecka, M., and Struzik, Z. R. (2015b). Entropic measures of complexity of short-term dynamics of nocturnal heartbeats in an aging population. Entropy 17, 1253-1272. doi:10. 3390/e17031253

Makowiec, D., Rynkiewicz, A., Galaska, R., Wdowczyk-Szulc, J., and ŻarczyńskaBuchowiecka, M. (2011). Reading multifractal spectra: aging by multifractal analysis of heart rate. Europhys. Lett. 94, 68005. doi:10.1209/0295-5075/94/ 68005

Makowiec, D., Struzik, Z. R., Graff, B., Żarczyńska-Buchowiecka, M., and Wdowczyk, J. (2014). Transition network entropy in characterization of

\section{Acknowledgments}

The authors DM, ZRS, DW, and AK acknowledge the financial support of the National Science Center, Poland, UMO: 2012/06/M/ST2/00480.

complexity of heart rhythm after heart transplantation. Acta Phys. Pol. Ser. B 45, 1771-1781. doi:10.5506/APhysPolB.45.1771

Martini, F. H., Ober, W. C., and Nath, J. L. (2011). Visual Anatomy \& Physiology. San Francisco: Pearson Benjamin Cummings.

Meersman, R. E. D., and Stein, P. K. (2007). Vagal modulation and aging. Biol. Psychol. 74, 165-173. doi:10.1016/j.biopsycho.2006.04.008

Monahan, K. D. (2007). Effect of aging on baroreflex function in humans. Am. J. Physiol. Regul. Integr. Comp. Physiol. 293, R3-R12. doi:10.1152/ajpregu.00031. 2007

Monti, A., Medigue, C., Nedelcoux, H., and Escourrou, P. (2002). Autonomic control of the cardiovascular system during sleep in normal subjects. Eur. J. Appl. Physiol. 87, 174-181. doi:10.1007/s00421-002-0597-1

Pikkujämsä, S. M., Mäkikallio, T. H., Sourander, L. B., Räihä, I. J., Puukka, P., Skyttä, J., et al. (1999). Cardiac interbeat interval dynamics from childhood to senescence: comparison of conventional and new measures based on fractals and chaos theory. Circulation 100, 393-399. doi:10.1161/01.CIR.100.4.393

Poirier, P. (2014). Exercise, heart rate variability, and longevity: the cocoon mystery? Circulation 129, 2085-2087. doi:10.1161/CIRCULATIONAHA.114.009778

Porta, A., Faes, L., Bari, V., Marchi, A., Bassani, T., Nollo, G., et al. (2014). Effect of age on complexity and causality of the cardiovascular control: comparison between model-based and model-free approaches. PLoS ONE 9:e89463. doi:10. 1371/journal.pone.0089463

Reardon, M., and Malik, M. (1996). Changes in heart rate variability with age. Pacing Clin. Electrophysiol. 19, 1863-1866. doi:10.1111/j.1540-8159.1996.tb03241.x

Schmitt, D. T., and Ivanov, P. C. (2007). Fractal scale-invariant and nonlinear properties of cardiac dynamics remain stable with advanced age: a new mechanistic picture of cardiac control in healthy elderly. Am. J. Physiol. Regul. Integr. Comp. Physiol. 293, R1923-R1937. doi:10.1152/ajpregu.00372.2007

Schmitt, D. T., Stain, P. K., and Ivanov, P. C. (2009). Stratification pattern of static and scale-invariant dynamic measures of heartbeat fluctuations across sleep stages in young and elderly. IEEE Trans. Biomed. Eng. 56, 1564-1573. doi:10.1109/TBME.2009.2014819

Schumann, A. Y., Bartsch, R. P., Penzel, T., Ivanov, P. C., and Kantelhardt, J. W. (2010). Aging effects on cardiac and respiratory dynamics in healthy subjects across sleep stages. Sleep 33, 943-955.

Shimazu, T., Tamurai, N., and Shimazu, K. (2005). Aging of the autonomic nervous system. Nihon Rinsho 63, 973-977.

Stein, P. K., Barzilay, J. I., Chaves, P. H. M., Domitrovich, P. P., and Gottdiener, J. S. (2009). Heart rate variability and its changes over 5 years in older adults. Age Ageing 38, 212-218. doi:10.1093/ageing/afn292

Stein, P. K., and Pu, Y. (2012). Heart rate variability, sleep and sleep disorders. Sleep Med. Rev. 16, 47-66. doi:10.1016/j.smrv.2011.02.005

Struzik, Z. R., Hayano, J., Sakata, S., Kwak, S., and Yamamoto, Y. (2004). 1/f scaling in heart rate requires antagonistic autonomic control. Phys. Rev. E Stat. Nonlin. Soft Matter Phys. 70, 050901. doi:10.1103/PhysRevE.70.050901

Struzik, Z. R., Hayano, J., Soma, R., Kwak, S., and Yamamoto, Y. (2006). Aging of complex heart rate dynamics. IEEE Trans. Biomed. Eng. 53, 89-94. doi:10.1109/ TBME.2005.859801

TaskForce. (1996). Task Force of the European Society of Cardiology, North American Society of Pacing. Heart rate variability: standards of measurement, physiological interpretation, and clinical use. Circulation 93, 1043-1065. doi:10. 1161/01.CIR.93.5.1043

Tobaldini, E., Nobili, L., Strada, S., Casali, K. R., Braghiroli, A., and Montano, N. (2013). Heart rate variability in normal and pathological sleep. Front. Physiol. 4:294. doi:10.3389/fphys.2013.00294

Trinder, J. (2008). Cardiovascular control during sleep: "Sleep-dependent changes in the coupling between heart period and blood pressure in human subjects," by Silvani et al. Am. J. Physiol. Regul. Integr. Comp. Physiol. 294, R1684-R1685. doi:10.1152/ajpregu.00187.2008

Viola, A. U., Tobaldini, E., Chellappa, S. L., Casali, K. R., Porta, A., and Montano, N. (2011). Short-term complexity of cardiac autonomic control during sleep: REM 
as a potential risk factor for cardiovascular system in aging. PLoS ONE 6:e19002. doi:10.1371/journal.pone.0019002

Conflict of Interest Statement: The authors declare that the research was conducted in the absence of any commercial or financial relationships that could be construed as a potential conflict of interest.
Copyright (c) 2015 Makowiec, Wejer, Kaczkowska, Żarczyńska-Buchowiecka and Struzik. This is an open-access article distributed under the terms of the Creative Commons Attribution License (CC BY). The use, distribution or reproduction in other forums is permitted, provided the original author(s) or licensor are credited and that the original publication in this journal is cited, in accordance with accepted academic practice. No use, distribution or reproduction is permitted which does not comply with these terms. 